\title{
The Effect of BP Measurement Velocity on Noninvasive BP Measurement Value Was Studied by Establishing the Blood Pressure Measurement Running Model
}

\author{
Du Xuelian, Wang Bingtao, Wang Baiyan \\ Beijing Electric Power Hospital, Beijing, China
}

\section{Email address:}

doudou720109@163.com (Du Xuelian)

\section{To cite this article:}

Du Xuelian, Wang Bingtao, Wang Baiyan. The Effect of BP Measurement Velocity on Noninvasive BP Measurement Value Was Studied by Establishing the Blood Pressure Measurement Running Model. Science Discovery. Vol. 7, No. 3, 2019, pp. 165-171.

doi: $10.11648 /$ j.sd.20190703.16

Received: April 11, 2019; Accepted: June 11, 2019; Published: June 15, 2019

\begin{abstract}
Objective Through the establishment of blood pressure measurement running model, the relationship between the blood pressure measurement velocity and the noninvasive blood pressure measurement value was calculated, then the accuracy of noninvasive blood pressure measurement was evaluated. Methods First, the basis of establishing the blood pressure measurement running model: the origin and nature of the blood pressure auscultation sound (BPAS) which was also confirmed as the main component of Korotkoff-sound was determined by the resonance theory of blood pressure measurement and its confirming experiments from the perspective of evidence-based medicine. The BPAS was a vibration sound which coming from the vibration of closing mitral valve and being homologous with the first heart sound and also possessing resonant nature. It was located between the first heart sound and the second heart sound on the synchronization graph of artery and heart, this meant that the Korotkoff-sound was a sound of systolic period in cardiac cycle; Second, establishing the blood pressure measurement running model: the cardiac cycle was divided into 6 equal parts in which 3 ones were in systolic period and another 3 ones were in diastolic period, then 6 pressure measurement lines were imagined and the speed that the gas flow out of the cuff was in control from $1-10 \mathrm{mmHg} /$ per beating and the systolic pressure measurement value was calculated using mathematical methods, the principle of value reading was to use round figure; In final, setting up the ideal tested individuals: imagining the individual's systolic pressure of each beating was always $130 \mathrm{mmHg}$, the heart rate was $60 / \mathrm{min}$. Results When the blood pressure measurement velocity was $1 \mathrm{mmHg} /$ per beating, two numbers of $130 \mathrm{and} 129 \mathrm{mmHg}$ were calculated; when the velocity was 2 to $3 \mathrm{mmHg} /$ per beating, three numbers of 130,129 and $128 \mathrm{mmHg}$ were calculated; when the velocity was 4 to $5 \mathrm{mmHg}$ /per beating, five numbers from 130 to $126 \mathrm{mmHg}$ were calculated; when the velocity was $10 \mathrm{mmHg} /$ per beating, nine numbers from 130 to $122 \mathrm{mmHg}$ were calculated; Conclusion The faster the blood pressure measurement velocity was, the wider the range of blood pressure measurement value was, and then the greater the measurement error was. The blood pressure measurement velocity can greatly affect the systolic blood pressure measurement value , and this is also an important factor of fluctuation in blood pressure.
\end{abstract}

Keywords: Blood Pressure Measurement Velocity, Blood Pressure Measurement Value, Blood Pressure Measurement Running Model, Blood Pressure Auscultation Sound, Resonance Theory, Phonoarteriogram, Phonocardiogram 


\title{
通过血压测量运行模型的建立研究测压速度对无创血压测量值的 影响
}

\section{杜雪莲, 王滨涛, 王柏岩}

北京电力医院疾控处，北京，中国

\section{邮箱}

doudou720109@163.com（杜雪莲）

\begin{abstract}
摘要：目的：建立血压测量运行模型并计算测压速度和无创血压测量值的关系, 评价无创血压测量的准确性。方 法: 1、模型建立的依据: 血压测量共振理论及实验从循证医学的角度确定了作为柯氏音主要组分的血压听诊音起 源于以二尖瓣为主的房室瓣关闭产生的振动, 具有共振的性质, 并与第一心音同源, 是收缩期声音; 2、建立血压 测量运行模拟图: 把脉音图上的每一个心动周期均分成6份, 收缩期与舒张期各占 3 份, 再虚拟 6 条测压线, 通过数 学的方法推算不同测压速度下收缩压的理论值 (取整数)； 3、设立理想被测血压个体：每次心搏收缩压均为 $130 \mathrm{mmHg}$, 心率是 60 次 $/ \mathrm{min}$ 。结果：测压速度是 $1 \mathrm{mmHg} /$ 每心搏时测出 130 和 $129 \mathrm{mmHg}$ 两个值; 测压速度为 2 或 $3 \mathrm{mmHg} /$ 每心搏时测出 130,129 和 $128 \mathrm{mmHg}$ 三个值; 测压速度是 4 和 $5 \mathrm{mmHg} /$ 每心搏时测出 130 至 $126 \mathrm{mmHg}$ 区间的 五个值; 测压速度是 $10 \mathrm{mmHg} /$ 每心搏时测出 130 至 $122 \mathrm{mmHg}$ 区间的九个值。结论: 测压速度越快, 测出的收缩压 值范围越广，误差越大。测压速度可以极大地影响收缩压的测量，是血压波动的重要因素。
\end{abstract}

关键词: 测压速度, 血压测量值, 血压测量运行模型, 血压听诊音, 共振理论, 脉音图, 心音图

\section{1. 引言}

无创血压测量中的影响因素大致可以分成四个方面 [1], 一是测量设备的因素, 如水银血压计和电子血压计的 测量结果是不同的; 二是操作者的因素, 如操作者的听力、 操作的熟练程度、测压速度等; 三是受试者的因素, 如血 压的波动性、受试者自身调件（胖瘦、上肢粗度）[2]; 四 是环境因素, 如温度、嘈杂程度, 是可控因素。故严格地 讲只有三方面影响因素。单一因素如何影响无创血压测量 值却无从知晓, 因为医学界一直没搞清楚血压测量的判 定标识----柯氏音（主要组分是敲击样声音的血压听诊音） 的起源和性质, 且人们只了解这些因素综合作用后对无创 血压测量值大致的影响[3]。我们提出了血压测量的共振理 论[4], 并且通过心音图的描记设备发现了脉音波形和血压 听诊音波形以及每次心搏的血压波动现象的存在, 从而验 证了血压测量的共振理论 [5-7], 这就使得探讨测压速度对 无创血压测量值的影响成为可能。

\section{2. 方法}

\section{1. 血压测量运行模拟图建立的依据}

血压测量的共振理论及其实验证实了作为柯氏音 主要成分的血压听诊音是起源于以二尖瓣为主的房室 瓣膜关闭所产生的振动（与第一心音同源），其在脉音 图上出现在主峰脉音和后峰脉音之间的位置, 多数呈直 线条形态, 占时极短而几乎无法测量其时长。脉音和心 音的同步图还证明主峰脉音和第一心音同源, 后峰脉音 和第二心音同源。据此确定：血压听诊音是上源性起源
的共振音, 不是局部起源的浴流音, 其位于脉音图的收 缩中期的位置。

\section{2. 血压听诊音分布模拟图}

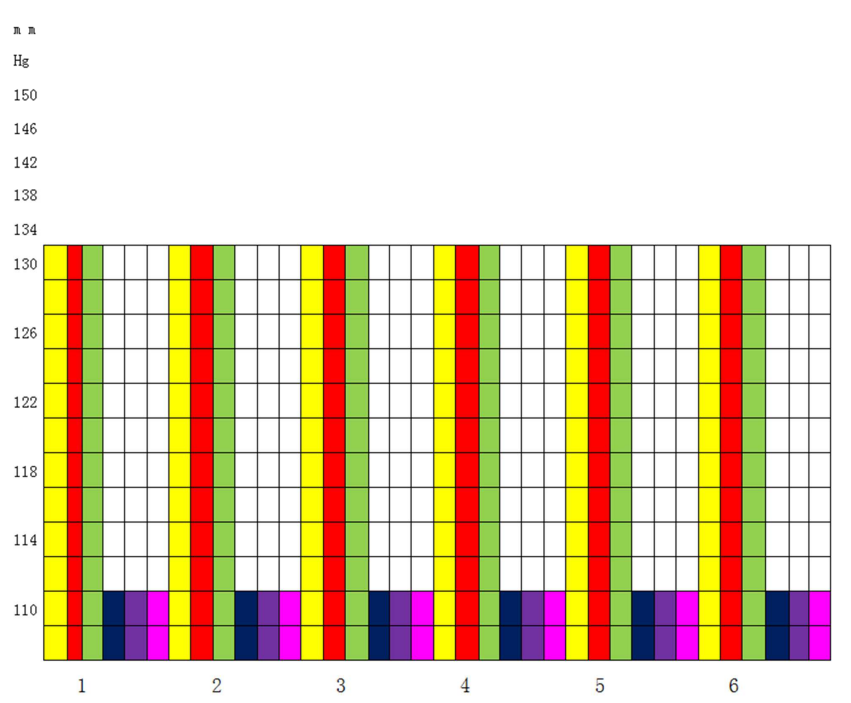

图1 血压听诊音分布模拟图。

横坐标数字代表第 $\mathrm{N}$ 次心搏, 纵坐标每格等于 $2 \mathrm{mmHg}$ (空白处每格等 于 $4 \mathrm{mmHg}$ )

一般认为, 心动周期的收缩期和舒张期之比为 $3: 5$, 但为了数学推算的简单化, 我们把整个心动周期均分成六 个时段, 以黄、红、绿三个高彩色柱组成收缩早、中、晚 期压力柱, 以黑、紫、粉三个矮彩色柱组成了舒张早、中、 晚期压力柱, 这样, 每个时段占时 0.167 秒, 大致相当于 第一心音约140-160毫秒的时长 [8], 也恰好相当于每分钟 
约 60 次心率时的六分之一心动周期时长。照此看来, 血压 听诊音正好落在收缩中期压力柱上, 这就是说, 只有收缩 中期才能读取血压（图中红色高彩色柱）。

\section{3. 血压测量运行模拟图}

在血压听诊音分布模拟图的基础上设计血压测量运 行模拟图。我们知道, 血压测量是一个盲测的过程, 测 试者不知道被测压个体大致的血压情况, 也不知道测量 的是哪一次心搏形成的血压, 更不知道给袖带放气时的 起始压力一定就高过被测压个体血压值的 20 或 $30 \mathrm{mmHg}$, 一切都是随机的。实际上, 即使测试者了解被测压个体 大致的血压情况, 也不能保证每次测压均以对应于心动
周期的同一个放气时点开始测压过程（比如每次都从收 缩压早期时点放气），并结束于同一个时点，这本身就 是测量误差的来源。分别以高过预计收缩压 $20 \mathrm{mmHg}$ 的 某次心搏的收缩早、中、晚期和舒张早、中、晚期 6 个部 位的起始时点为放气起点, 与各自对应的另一次心搏的 同一位置的起始时点为终点, 以测压速度分别为 $1-10 \mathrm{mmHg} /$ 每心搏时各自画一条线共画出6条平行的斜 线 (称虚拟测压线)，会相交于后续的某几个相关的心 搏形成的 6 个压力柱上。按照无创血压测量法的要求, 出 现第一个血压听诊音时读取血压计上的水银柱高度, 此 为收缩压, 在模拟图上就是第一个与虚拟测压线相交的 红色高彩色柱。

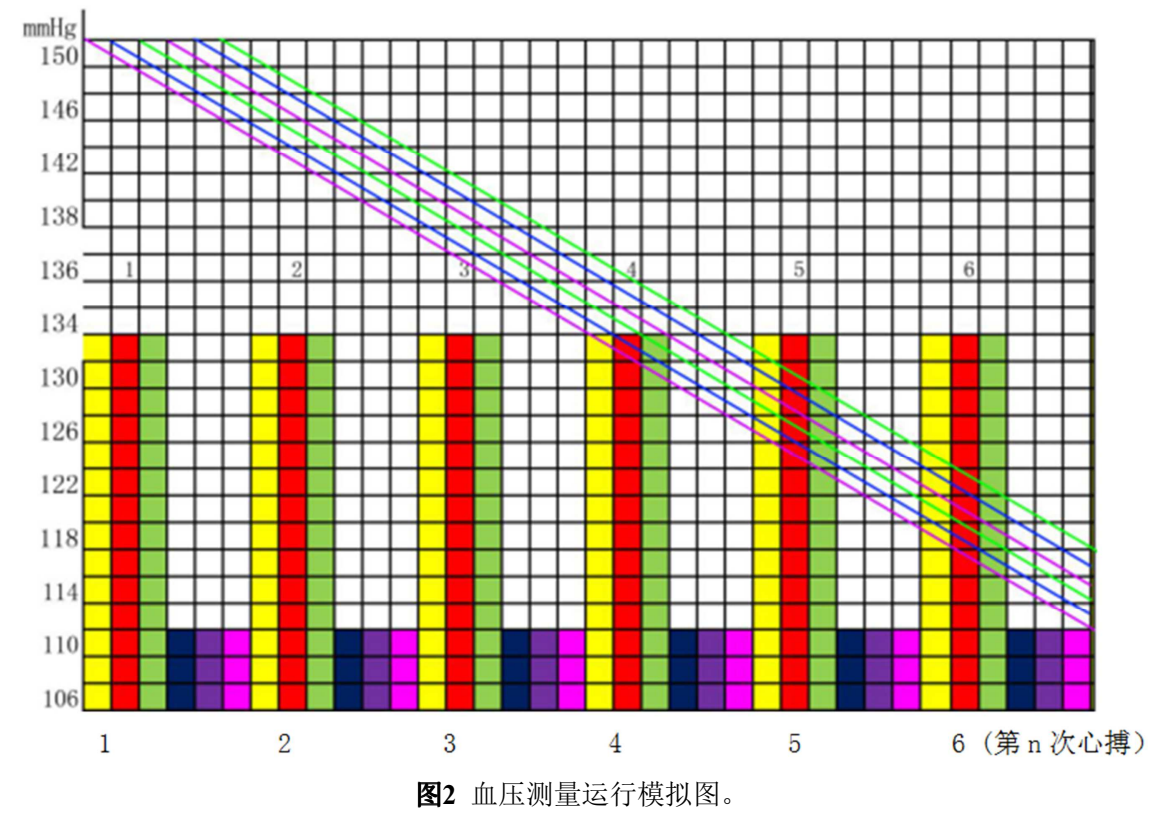

收缩压为 $130 \mathrm{mmHg}$, 舒张期为 $94 \mathrm{mmHg}$ 。起始放气压力 $150 \mathrm{mmHg}$, 以恒定的 $7 \mathrm{mmHg} /$ 每心搏的测压速度匀速放气, 形成了斜率 (即测压速度) 为 $7 \mathrm{mmHg} /$ 每心搏的6条虚拟测压线。

\section{4. 设立理想被测压个体}

假定被测压个体处在理想状态下，即不存在血压的波 动 (每次心搏形成的血压是恒定的), 那么, 用同一台测 压设备, 同一个操作者, 针对同一个被测压者测得的无创 血压值的唯一影响因素就是测压速度了。经过复杂的演算, 我们推导出用于 6 条虚拟测压线的理想状态下的测压计算 公式。6条虚拟测压线测压起点的起始压力都是 $150 \mathrm{mmHg}$, 测压速度可以是 $1 、 2 、 3 、 4 、 5 、 6 、 7 、 8 、 9 、 10 \mathrm{mmHg} /$ 每心搏的任意整数值, 读数原则是按四舍五入取整数 (因 为水银血压计最大分辨率是 $1 \mathrm{mmHg}$ ）。

\section{5. 计算公式}

被测压个体的收缩压为 $130 \mathrm{mmHg}$, 舒张期为 $94 \mathrm{mmHg}$ 。 起始放气压力 $150 \mathrm{mmHg}$, 如果以恒定的 $7 \mathrm{mmHg} /$ 每心搏的 测压速度匀速放气, 形成了斜率 (以及测压速度) 为 $7 \mathrm{mmHg} /$ 每心搏的 6 条虚拟测压线 (图2)。

测压公式: $\mathrm{vSn}=150-\mathrm{v} *(\mathrm{~N}-1) \pm \mathrm{v} * \mathrm{~B} / 6$,

$\mathrm{v}$ 表示测压速度为 $1-10 \mathrm{mmHg}$ 的任意值,
$S$ 为测压值,

$\mathrm{n}$ 表示第 1 至第 6 条虚拟测压线,

$\mathrm{v}$ : 测压速度,

$\mathrm{N}$ : 与测压线相交的某次心搏,

B：时段数(共 6 个时段, 提前交于红色压力柱上的时 段数用减号, 延迟交于红色压力柱上的时段数用加号, 交 于下一次心搏的时段数亦用减号）。

\section{6. 数据推导过程}

当起始测压压力是 $150 \mathrm{mmHg}$ (即过加压压力是 $20 \mathrm{mmHg}$ ）时, 则测出收缩压值的心搏一定是第 21 次或 22 次, 那么:

(1)此速度的收缩早期测压线（1S1）运行到第21次心 搏刚好交于收缩早期压力柱上, 其袖带内压力值刚好是 $150-1 *$ * (21-1) $=130 \mathrm{mmHg}$, 但因为该期并没有血压听诊 音出现, 故不能判定收缩压值, 需继续运行 1 个时段 (用 减号) 才交到收缩中期的红色高彩色柱 (亦称血压听诊音 柱）上, 音压匹配的袖带内压力值是: $1 \mathrm{~S} 1=150-1 *$ (21-1) $-1 * 1 / 6=129.83$, 取整数收缩压为 $130 \mathrm{mmHg}$; 
(2)此速度的收缩中期测压线（1S2）运行到第21次心 搏的血压听诊音柱上正好满 20 个心动周期, 音压匹配的袖 带内压力值为: $1 \mathrm{~S} 2=150-1 *(21-1)=130.00$, 取整数收缩压 为 $130 \mathrm{mmHg}$;

(3)此速度的收缩晚期测压线（1S3）运行到第21次心 搏的血压听诊音柱上正好是差 1 个时段（用加号）满 20 个 心动周期, 袖带内压力值高于理想血压值, 仍不能读取袖 带内压力值, 需继续运行至下一次心搏 (即第22次心搏) 的血压听诊音柱上, 则多运行了 5 个时段 (用减号), 此 时音压匹配的袖带内压力值为: $1 \mathrm{~S} 3=150-1 *(21-1)-1 * 5 / 6=129.17$, 取整数收缩压为 $129 \mathrm{mmHg}$;

(4)此速度的舒张早期测压线（1S4）运行到第21次心 搏的收缩早期压力柱上时 (不满 20 个心动周期) 压力高于 理想血压值, 同(3)类似, 满 20 个心动周期后需继续运行 4 个时段（用减号）交于下一次心搏（第22次），此时音压 匹配的袖带内压力值为: $1 \mathrm{~S} 4=150-1 *(21-1)-1 * 4 / 6=129.33$, 取整数收缩压为 $129 \mathrm{mmHg}$;

(5)此速度的舒张中期测压线（1S5）运行到第21次心 搏的收缩早期压力柱上时 (不满 20 个心动周期) 压力高于
理想血压值, 同(3)类似, 满 20 个心动周期后需继续运行 3 个时段（用减号）交于下一次心搏（第22次），此时音压 匹配的袖带内压力值为: $1 \mathrm{~S} 5=150-1 *(21-1)-1 * 3 / 6=129.50$, 取整数收缩压为 $130 \mathrm{mmHg}$;

(6)此速度的舒张晚期测压线（1S6）运行到第21次心 搏的黄色柱上时 (不满 20 个心动周期) 压力高于理想血压 值, 满 20 个心动周期后需继续运行 2 个时段 (用减号) 交 于下一次心搏（第22次）, 同(3)类似, 此时音压匹配的袖 带内压力值为: $1 \mathrm{~S} 6=150-1 *(21-1)-1 * 2 / 6=129.67$, 取整数 收缩压为 $130 \mathrm{mmHg}$ 。

\section{3. 结果}

\section{1. 当被测压个体的理想收缩压为 $130 \mathrm{mmHg}$ 时的推算结 果}

因为数学计算占篇幅太大, 本文只演示了测压速度为 $1 \mathrm{mmHg} /$ 每心搏的计算过程。全部 $1-10 \mathrm{mmHg} /$ 每心搏的测 压速度的计算结果列表如下:

表 1 测压速度为 $1 \mathrm{mmHg}$ 时。

\begin{tabular}{|c|c|c|c|c|c|c|c|}
\hline 理想血压 & 1 s1 & $1 \mathrm{~s} 2$ & $1 \mathrm{~s} 3$ & $1 \mathrm{~s} 4$ & $1 \mathrm{~s} 5$ & 156 & 均值 \\
\hline $130 \mathrm{mmHg}$ 时 & $129.83(130)$ & $130.00(130)$ & $129.17(129)$ & $129.33(129)$ & $129.50(130)$ & $129.67(130)$ & 129.58 \\
\hline 理想血压 & $2 \mathrm{~s} 1$ & $2 \mathrm{~s} 2$ & $2 \mathrm{~s} 3$ & $2 \mathrm{~s} 4$ & $2 s 5$ & $2 \mathrm{~s} 6$ & 均值 \\
\hline $130 \mathrm{mmHg}$ 时 & $129.67(130)$ & $130.00(130)$ & $128.33(128)$ & $128.67(129)$ & $129.00(129)$ & $129.33(129)$ & 129.17 \\
\hline
\end{tabular}

表3 测压速度为 $3 \mathrm{mmHg}$ 时。

\begin{tabular}{llllllll}
\hline 理想血压 & 3s1 & 3s2 & 3s3 & 3s4 & 3s5 & 3s6 & 均值 \\
\hline $130 \mathrm{mmHg}$ ( 3 & $128.50(129)$ & $129.00(129)$ & $129.50(130)$ & $130.00(130)$ & $127.50(128)$ & $128.00(128)$ & 128.75 \\
\hline
\end{tabular}

表4 测压速度为 $4 \mathrm{mmHg}$ 时。

\begin{tabular}{llllllll}
\hline 理想血压 & 4s1 & 4s2 & 4s3 & 4s4 & 4s5 & 4s6 & 均值 \\
\hline $130 \mathrm{mmHg} \mathbf{3}$ & $129.33(129)$ & $130.00(130)$ & $125.83(126)$ & $127.33(127)$ & $128.00(128)$ & $128.67(129)$ & 128.45 \\
\hline
\end{tabular}

表 5 测压速度为 $5 \mathrm{mmHg}$ 时。

\begin{tabular}{llllllll}
\hline 理想血压 & $\mathbf{5 s 1}$ & $\mathbf{5 s 2}$ & $\mathbf{5 s 3}$ & $\mathbf{5 s 4}$ & $\mathbf{5 s 5}$ & $\mathbf{5 s 6}$ & 均值 \\
\hline $130 \mathrm{mmHg}$ ( $\mathbf{3}$ & $129.83(130)$ & $130.00(130)$ & $125.83(126)$ & $126.67(127)$ & $127.50(128)$ & $128.33(128)$ & 128.03 \\
\hline
\end{tabular}

表6 测压速度为 $6 \mathrm{mmHg}$ 时。

\begin{tabular}{llllllll}
\hline 理想血压 & $\mathbf{6 s 1}$ & $\mathbf{6 s 2}$ & $\mathbf{6 s 3}$ & $\mathbf{6 s 4}$ & $\mathbf{6 s 5}$ & $\mathbf{6 s 6}$ & 均值 \\
\hline $130 \mathrm{mmHg} \mathbf{3}$ & $125.00(125)$ & $126.00(126)$ & $127.00(127)$ & $128.00(128)$ & $129.00(129)$ & $130.00(130)$ & 127.50 \\
\hline
\end{tabular}

表7 测压速度为 $7 \mathrm{mmHg}$ 时。

\begin{tabular}{llllllll}
\hline 理想血压 & $\mathbf{7 s 1}$ & $\mathbf{7 s 2}$ & $\mathbf{7 s 3}$ & $\mathbf{7 s 4}$ & $\mathbf{7 s 5}$ & $\mathbf{7 s 6}$ & 均值 \\
\hline $130 \mathrm{mmHg} \mathbf{3}$ & $127.83(128)$ & $129.00(129)$ & $130.00(130)$ & $124.33(124)$ & $125.50(126)$ & $126.67(127)$ & 127.22 \\
\hline
\end{tabular}

表8 测压速度为 $8 \mathrm{mmHg}$ 时。

\begin{tabular}{llllllll}
\hline 理想血压 & $\mathbf{8 s 1}$ & $\mathbf{8 s 2}$ & $\mathbf{8 s 3}$ & $\mathbf{8 s 4}$ & $\mathbf{8 s 5}$ & $\mathbf{8 s 6}$ & 均值 \\
\hline $130 \mathrm{mmHg} \mathbf{3}$ & $124.67(125)$ & $126.00(126)$ & $127.33(127)$ & $128.67(129)$ & $130.00(130)$ & $123.33(123)$ & 126.66 \\
\hline
\end{tabular}


表9 测压速度为 $9 \mathrm{mmHg}$ 时。

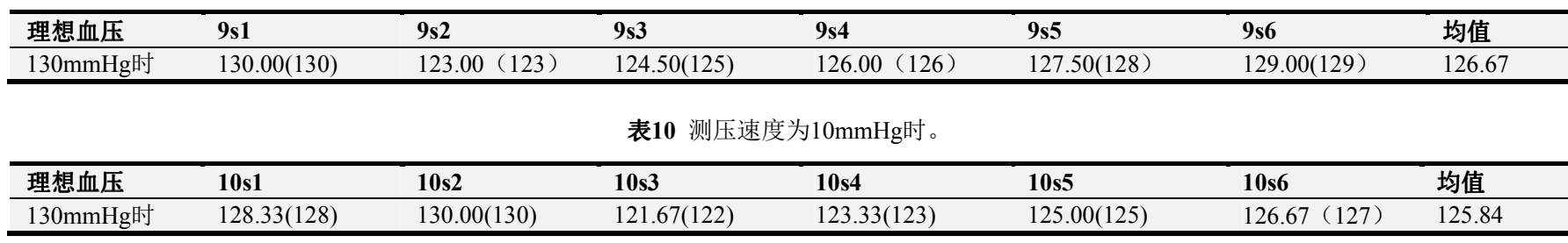

\section{2. 当被测压个体的理想收缩压为其它数值时的推算结 果}

对于被测压个体的理想收缩压值为其它数值 $\mathrm{x}$ 时, 读 取收缩压值的心搏位置会发生改变, 或提前或延后, 读出 的收缩压值在 $130 \mathrm{mmHg}$ 的理想血压值推算的基础上再加 上x-130即可。

理想血压为 $x$ 时推算的测压结果 $=$ 某一测压速度下理 想血压为 $130 \mathrm{mmHg}$ 的推算测量值 $+\{\mathrm{x}-130\}$

\section{4. 讨论}

\section{1. 无创血压测量法的对与错}

我们认为, 医学界尚缺乏对无创血压测量法的评价, 包括两方面: 一是有关对与不对的问题, 二是有关准与不 准的问题。血压测量的共振理论及其实验已经从循证医学 的角度解决了第一个问题, 其认为对无创收缩压的测量是 对的, 但对无创舒张压的测量是错的, 其中的循证证据就 是在实验中发现了作为柯氏音的主要组成成分的具有敲 击样声音特点的，同时也是柯氏音 1 期唯一组成成分的血 压听诊音只出现在脉音和心音同步图上心动周期的收缩 期内, 从未出现在舒张期内, 即用出现在收缩期的血压测 量判定标识判定收缩压是对的, 而同时用它判定舒张压则 是错的。实际上, 在柯氏医生之前的意大利罗西医生等前 辈从未用无创血压测量法测量过无创舒张压 [9]。既然无创 舒张压的测量是错的, 其自然也就不存在准不准的问题了, 但要彻底根除既往对所谓的无创舒张压的错误认识尚需 时日。依据共振理论及其实验的观点, 人们测得的所谓的 无创收缩压和无创舒张压的科学称谓应该分别是最大共 振压和最小共振压 [4]。依据循证医学的要求, 必须要有确 丵的证据证明最大共振压就是无创收缩压, 而最小共振压 就是无创舒张压才行。共振理论及其实验肯定了最大共振 压就是无创收缩压, 但否定了最小共振压就是无创舒张压。 从所谓的无创舒张压测量的角度来看, 2590例研究对象的
有创舒张压和无创舒张压互为高低, 没有任何规律可言: 就一组正常人的测量数据而言, 男大学生所谓的无创舒张 压高于有创舒张压, 但女大学生所谓的无创舒张压反而低 于有创舒张压 [12]; 而一些包括重症高血压在内的病人其 所谓的无创舒张压也高于有创舒张压 $[27,45]$; 再有, 休 克病人所谓的无创舒张压却明显高于有创舒张压 $[10,12$, 19], 这些数据都不支持无创舒张压的测量。虽然否定了 无创舒张压的测量, 但共振理论及其实验认为最小共振压 即所谓的无创舒张压却代表着动脉硬化的程度: 此值越小 说明动脉硬化越严重。

\section{2. 无创收缩压测量值的准确性}

\subsection{1. 模型的推导结论与既往研究的佐证}

从依据共振理论建立的血压测量运行模型推导的测 压值的角度看, 无创收缩压是小于有创舒张压的。我们对 搜集到的36篇已发表的有创和无创血压的对照研究文章 中共2590例汇总分析发现[10-45]: 正常人群、高血压人群、 一般性疾病人群、贞幼儿人群、甚至包括心脏介入术人群 其无创收缩压全部小于有创收缩压, 由于此类人群占总人 群的绝大多数, 故此结论支持无创收缩压测量的正确性, 但无创收缩压明显低于有创收缩压达7 18mmHg之多[12, $31,40,45]$, 这说明无创收缩压的测量是不准确的; 在 休克病人更是反向差值, 即无创收缩压明显高于有创收缩 压达 $17-26 \mathrm{mmHg}$ 之多 $[10,19]$, 说明无创血压测量法的应用 是有其局限性的, 用于休克的诊断和监测会带来严重误导, 实际上也已经误导了临床多年。

\subsection{2. 从测压速度看无创收缩压测量的准确性}

临床上有创收缩压和无创收缩压的差值是综合因素 作用的结果, 而依据血压测量的共振理论及其实验建立的 血压测量运行模型, 旨在研究单一因素对血压测量的影响。 整理上述计算结果并重新列表如下:

表11 从测压速度看无创收缩压测量的准确性。

\begin{tabular}{|c|c|c|c|c|}
\hline 测压速度为 & 理想收缩压值 & 读数范围及最小读数 & 读数数量 & 读取收缩压值的第N次心搏 \\
\hline $1 \mathrm{mmHg}$ & 130 & $130-129$ & 2 & 21 \\
\hline $2 \mathrm{mmHg}$ & 130 & $130-128$ & 3 & $11 、 12$ \\
\hline $3 \mathrm{mmHg}$ & 130 & $130-128$ & 3 & $8 、 9$ \\
\hline $4 \mathrm{mmHg}$ & 130 & $130-126$ & 5 & $6 、 7$ \\
\hline $5 \mathrm{mmHg}$ & 130 & $130-126$ & 5 & 5,6 \\
\hline $6 \mathrm{mmHg}$ & 130 & $130-125$ & 6 & 5,6 \\
\hline $7 \mathrm{mmHg}$ & 130 & $130-124$ & 7 & $4 、 5$ \\
\hline $8 \mathrm{mmHg}$ & 130 & $130-123$ & 8 & $4 、 5$ \\
\hline $9 \mathrm{mmHg}$ & 130 & $130-123$ & 8 & 3,4 \\
\hline $10 \mathrm{mmHg}$ & 130 & $130-122$ & 9 & 3,4 \\
\hline
\end{tabular}


首先, 无创血压测量法测得的血压不会高于该个体真 实的血压, 这就是为什么一些血压正常的人也会发生相当 多的心脑血管事件的原因, 即血压不是不高, 而是没发现 血压高; 其次, 同一个测压速度可以测量出不高于该个体 理想血压值至少 2 个收缩压值, 且测压速度越快, 测量出 的收缩压范围越多, 测出的最低血压值越小, 即测量的结 果越不准, 这也是导致血压波动的一个重要原因。如表 11 中理想血压为 $130 \mathrm{mmHg}$ 的个体, $1 \mathrm{mmHg}$ 的测压速度时, 可以测量出 130 和 129 共 2 个整数收缩压值, 最多低测 $1 \mathrm{mmHg}$, 而当测压速度达到 $10 \mathrm{mmHg}$ 时却可以测量出 130-122之间共 9 个整数收缩压值, 最多低测了 $8 \mathrm{mmHg}$ 的收 缩压, 测压速度本身造成的血压波动性可见一斑。测压速 度是 $1 \mathrm{mmHg}$ 时产生的 $1 \mathrm{mmHg}$ 的测量误差对临床诊断和疗 效评价产生的影响很小, 即使是按照教科书规定的 $2-3 \mathrm{mmHg}$ /秒 (以每次心搏来确定测压速度更具操作性), 至多会出现 $3 \mathrm{mmHg}$ 的测量误差, 这也是可以接受的。就 临床实用性来讲, $1 \mathrm{mmHg}$ 的测压速度太慢, $2 \sim 3 \mathrm{mmHg}$ 的测压速度是比较合适的, 但更快的测压速度会导致更大 的无创血压测量误差, 故不建议采纳。

临床上, 如果我们测得了某一个体的收缩压值, 可以 明确该个体的收缩压值不会小于此值, 但小多少却不得而 知。水银柱式或气压表式血压计可以知道测得的是哪一次 心搏的收缩压，而大部分的电子血压计是通过脉搏波波形 的变化感知的血压, 不能肯定是哪一次心搏的收缩压。因 此, 试图准确测量血压, 或比较不同测压设备的血压测量 结果甚至搞清楚血压测量的误差完全是不可能的, 也是徒 劳的。只有同一次心搏的有创和无创血压同步研究才更合 乎科学要求。

\section{5. 结论}

血压测量的共振理论及其实验确定了无创血压测量 法对无创收缩压测量的科学性, 并否定了对无创舒张压测 量的科学性, 而依据其建立的血压测量运行模拟图, 则清 晰地阐明了测压速度和测量误差的关系: 理想被测压个体 其测压速度越快对收缩压测量值产生的误差越大。现实中 并不存在这样的理想被测压个体，那么，考虑到真实的被 测压个体极大的血压波动性, 测压速度对测压结果的影响 程度会更大, 测量误差更不好预测。因此, 放慢测压速度 是目前唯一可行的减少无创收缩压测量误差的办法。

\section{参考文献}

[1] 张旭芳, 焦守风, 韩艳.影响血压测量值误差原因分析.中国 误诊学杂志, 2011，11（16）:3902-3903.

[2] 董静, 任丽洁, 崔福江.血压测量结果误差原因分析.卫生职 业教育, 2015,33（13）：102-103.

[3] 王海燕, 柴萍. 120例患者应用三种血压计的测量结果及分 析.临床研究, 2015,23 (12) : 79-80.
[4] 王滨涛, 王柏岩.血压听诊音性质的理论探讨 [J].中国卫生产 业,2015,12（23）:9-12.

[5] 王滨涛, 王柏岩.血压测量共振理论的实验验证 (一) : 动 脉脉音图特点及波形命名.中华高血压杂志 2017,25(1):69-74.

[6] 王滨涛, 王柏岩.血压测量共振理论的实验验证（二）：动 脉脉音和血压听诊音的起源。中华高血压杂志 2017,25(2):157-162.

[7] 王滨涛, 王柏岩.血压测量共振理论的实验验证（三）：血 压听诊音的性质.中华高血压杂志2017,25(3):258-263.

[8] 刘大为. 临床血流动力学 $[\mathrm{M}]$.北京: 人民卫生出版社, 2013: 19.

[9] Zanchetti, Alberto;Mancia,Giuseppe. The centenary of blood pressure measurement: a tribute to Scipione Riva-Rocci. Journal of Hypertension. 1996, 14(1):1-12.

[10] 来芬华, 金琦.40例休克患者应用有创血压与无创血压监测 的比较.护理与康复, 2008,7 (4) : 288-289.

[11] 蔡丽萍, 王警卫, 陈晓茜等。无创和有创血压监测在手术 中的应用.武警医学院学报, 2008,17（5）：443-444.

[12] 陈业群, 潘红星。高校学生无创与有创血压的对比.汕头大 学医学院学报, 2012,25 (2) : 101-107.

[13] 邹春莉, 何义平, 于瑞英等。高血压危象病人无创血压与 有创血压测量差别的对比研究.实用护理杂志, 2003,19(7): $1-2$.

[14] 刘丽萍, 陈建昌, 徐卫亭。有创性血压和无创性血压的对 比分析.中国误诊学杂志, 2007,7(24):5738-5739.

[15] 司徒敏雄，马敬香, 余家康。危重症婴幼儿有创血压与无 创血压监测临床价值对比分析.中国实用儿科杂志, 2004,19 (7) : 438.

[16] 陈洪夏, 高传玉。心脏介入治疗时有创血压与无创血压监 测对比分析.中国误诊学杂志, 2006,6（6）：1093-1094.

[17] 潘英梅。有创动脉血压监测在ICU危重病人中的护理.中外 医疗, 2014,11:176-177.

[18] 方建中, 柳国胜, 吴立文等.有创及无创血压监测在新生儿 重症监护的应用对比研究.中国新生儿科杂志, 2013,28(4) 252-255.

[19] 宋林萍, 张星火, 顾秀丽等.97例休克患者有创血压与无创 血压监测的相关性研究.中华护理杂志, 2000,35（5）: 302-303.

[20] 宋稳丹.有创血压与无创血压在心脏手术后血压监测方面 的对比分析.吉林医学, 2011,32 (27) : 5665 .

[21] 曹权, 张萍, 宋燕波等.有创与无创血压监测分析.临床麻醉 学杂志, 2005,21 (8) : 571-572.

[22] 刘春莲.心脏术后早期有创血压与无创血压监测的对比分 析.医学信息2010,5 (11) :3131-3132. 
[23] 姚维红.有创血压和无创血压测量值的比较.现代中西医结 合杂志, 2007,16 (31):4643.

[24] 任春霞, 姜小鹰.心内直视术后有创血压与无创血压监测的 对照研究.护理研究, 2006, 20（8）：2009-2010.

[25] 张国强, 倪锦, 王怀贞等.贞幼儿无创和有创动脉血压对比 研究.国际医药卫生导报, 2006,12（12）：26-27.

[26] 林小红.休克早期患者有创和无创血压监测的比较.护士进 修杂志，2008,23（3）：265-266.

[27] 李宙, 王首红, 郭伟新.老年重症患者无创与有创血压测量 的比较.中国老年学杂志, 2009,29:1738-1739.

[28] 陈玉兰, 谢碧, 郭晓川.危重患者有创血压与无创血压监测 的比较.内蒙古中医药, 2013,22:137.

[29] 胡星兰, 卜晓星, 袁春风.成人有创血压及无创血压测量值 的比较.实用临床医学, 2006,7（8）：149-151.

[30] 谢谦.60例心脏外科术后有创血压与无创血压监测的对比 分析.中国医药指南，2013,11（34）：174-175. 13

[31] 邹春莉, 何义平, 李飞娥等.高血压病人无创血压值与有创 血压值的对比研究. 护理学杂志, 2003,18 (4) : 248-250.

[32] 魏化冰, 刘培娟, 黄影华. 人工机械瓣置换术后有创血压与 无创血压监测的对照研究. 中国实用护理杂志, 2009,25(4): 22-23. x

[33] 张金凤.90例心脏外科术后有创血压与无创血压监测结果 的对比.医学信息, 2014,27 (10) : 323 .

[34] 张金鍂, 皮红英. 有创血压监测与无创血压监测相关性分析. 中华现代护理杂志，2013,19（11）：1275-1277.
[35] 王晓玲.重症心脏病人术后有创血压与无创血压监测的对 比性探讨.安徽医学, 2004,25（4）：339-340.

[36] 詹金.体外循环术后有创血压与无创血压的比较.中国现代 药物应用，2010,4（20）：175-176.

[37] 陈媚, 高叶青.休克患者有创血压与无创血压产生差异的原 因分析.医学理论与实践, 2008,21（12）：1407-1408.

[38] 石绍南, 李向阳, 王美艳.患儿有创血压与无创血压的相关 性研究.中国医药指南, 2004, 2（12）：961-962.

[39] 向峦.冠状动脉旁路移植术后患者有创血压与无创血压的 对比研究.河北医药，2011,33（10）：1483-1484.

[40] 丁振江, 单伟超, 张英等.不同臂围有创血压与无创血压测 量结果的对比分析.承德医学院学报, 2013,30 (5):389-391.

[41] 李潇丹, 曲彦.上肢有创血压与无创血压的差异分析.中国医 药科学, 2012,2 (23) : 18-20.

[42] 朱健, 蔡慧芬. 有创及无创血压监测在休克患者中的应用 对比.现代中西医结合杂志, 2007,16（4）：492.

[43] 田宝石, 郭旋, 陈丽哲等。昏迷患者监测有创血压与无创 血压的临床意义.临床误诊误治, 2008,21（11）：31-32

[44] 王凤.麻醉后监测治疗室有创血压与无创血压相关性的研 究.中国护理管理, 2014,14增刊: 18-19

[45] 俞晓微, 丁振江, 赵洁等. 老年人主动脉有创血压测量与肱 动脉无创血压测量对比研究. 河北医药, 2011,33(8):1161-1162. 